\title{
DEMONSTRATION OF VIDEO-RATE DIFFUSE OPTICAL TOMOGRAPHY IN PHANTOMS AND TISSUES
}

\author{
Brian W. Pogue ${ }^{1 *}$, Daqing Piao ${ }^{2}$, Hamid Dehghani $^{1,3}$, Keith D. Paulsen ${ }^{1}$ \\ ${ }^{1}$ Thayer School of Engineering, Dartmouth College, Hanover NH 03755. \\ ${ }^{2}$ Department of Electrical Engineering, Oklahoma State University, Stillwater OK. \\ ${ }^{3}$ School of Physics, University of Exeter, Exeter UK. \\ * Email: pogue@dartmouth.edu
}

\begin{abstract}
Near-infrared diffuse optical tomography has been demonstrated with video rate acquisition of the transmitted signal for 8 sources and 8 detectors. The system design is outlined with components illustrated, and tomographic images are shown for phantoms and tissues. The system uses spectral encoding of the laser sources at small wavelength increments to allow each source location to be discerned at the detector. The key to this is spectrally dispersing the different wavelengths prior to detection, with a video rate CCD. Possible uses for this type of system are in the area of physiological monitoring and contrast agent kinetic imaging.
\end{abstract}

\section{INTRODUCTION}

Near-infrared imaging with diffuse light in tomography mode has been examined for a couple of decades. Only recently has there been a focus on developing fast imaging systems, as much of the attention has focused on biological applications which did not require fast imaging in tomographic mode. Fast imaging in remission mode has been a stable of functional activation studies, yet in tomographic mode, the ability to resolve location has been problematic, because the resolution of diffuse tomography is quite limited. However in recent years, the marriage of diffuse tomography with ultrasound [1], tomosynthesis [2] and MRI [3-5] have shown that diffuse tomography may not be limited by the low resolution limitation of the inverse image reconstruction problem. Rather, it is possible to couple such systems into the standard clinical imaging modalities and utilize their superior localization capabilities, and then provide NIR spectral information about those localized regions.

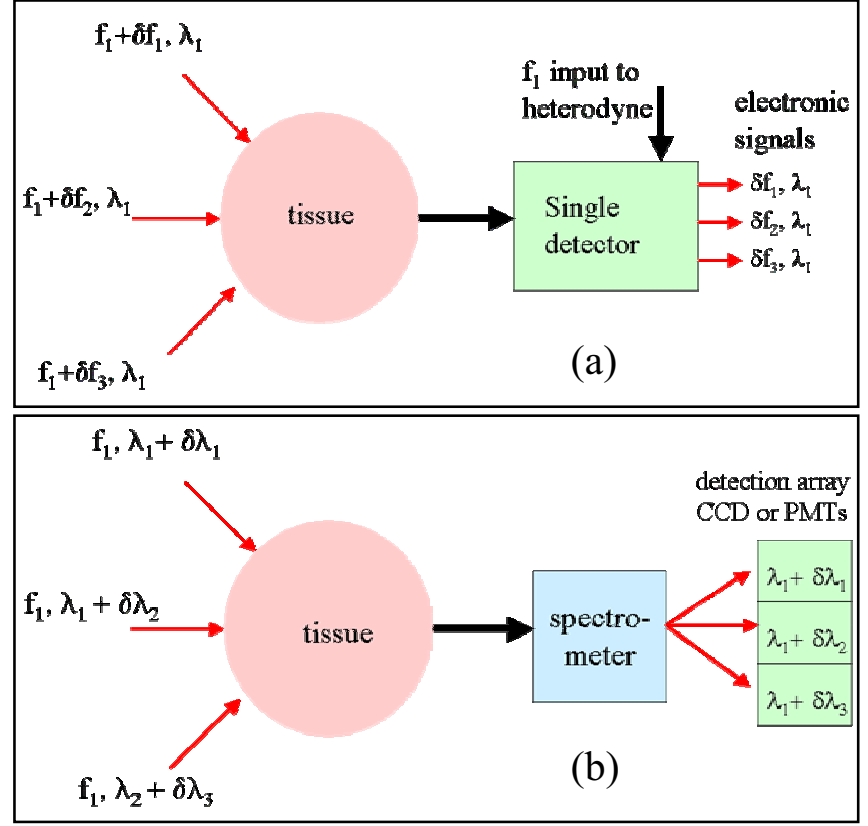

Figure 1. Graphical representation of two methods to parallelize the source locations (smaller red input arrows) to be measured at a single detector location (larger black output arrow). In (a) different heterodyne frequencies are used to encode the multiple source locations, which can then be detected simultaneously with one detector and the signal mixed with the original carrier frequency, $f$. Then each source signal is extracted from the Fourier transform of the data, where the location of the source is encoded by $\delta \mathrm{f}_{1}, \delta \mathrm{f}_{2}, \delta \mathrm{f}_{3}$. This approach has the inherent limitation that each additional source reduces the dynamic range of the detector, and hence decreases the signal to noise ratio. In (b), the spectral encoding of the sources is accomplished by offsetting the wavelength by small amounts, $\delta \lambda_{1}, \delta \lambda_{2}, \delta \lambda_{3}$, which are sufficient to allow signal separation at the output by using a high resolution spectrometer. The detection of the signal can still be done in frequency domain if coupled to multiple fast PMTs.

In this abstract, we discuss one aspect of an advanced tomography system, namely the ability to design a system which achieves video frame rate [6,7], similar to an ultrasound system, whereby tomographic data and images are provided by having parallel source and parallel detection simultaneously. There are several configurations which would possibly allow this type of detection, including 
frequency-encoding of the light sources, as introduced by Fraceschini et al [8] and illustrated in Figure 1(a), but in this abstract we demonstrate the concept of wavelength encoding of the light source, as illustrated in Figure 1(b). This approach is implemented in hardware and demonstrated for its utility in both phantom studies and tissue studies.

\section{METHODS}

The system was constructed from components purchased from electronics and laser/optics suppliers, as described in a previous paper [7]. The essence of the system was eight diode lasers all working at the same nominal wavelength band, but being tuned to slightly offset wavelengths by approximately $1.4 \mathrm{~nm}$ each, through temperature and power control of their set points. The lasers were introduced to fibers and launched into the tissue at 8 equally spaced locations in a circle. A series of 8 pick up fibers were placed also equally spaced around the circular region, to deliver remitted light to the spectrometer/CCD detector. At the spectrometer end, all fibers were placed in a vertical line, aligned with the spectrometer entrance slit, and the light was spread laterally through the spectrometer to separate each of the different wavelength bands at the CCD surface. This configuration allowed detection of all 8 source light signals at all 8 detector points in parallel, with the only limitation on acquisition being the frame rate of the CCD and the input light intensity.

\section{RESULTS}

Wavelength measurements were taken with the system, and the driving current and temperature settings were adjusted to provide equally spaced wavelengths within the 776 to $787 \mathrm{~nm}$ wavelength band. This is illustrated in the intensity graph of Figure 3(a). A reference sample from each laser was taken with a prism, immediately at the output of each laser, and these were all fed into the spectrometer at a single fiber location in the bottom to allow for control of the laser intensities. The transmitted intensities were measured by streaming the CCD camera to files which could be read in post processing and intensities binned in a MATLAB program to provide accurate measurements.

The crosstalk between channels was estimated to be below detectable levels, and the major problem limiting the dynamic range was simply the fact that the camera was not gated nor shuttered during operation, so there was vertical streaking of the image during the readout time of the imaging. This streaking was $1.7 \%$ of the maximal intensity, but was static throughout most image frames, so it could be largely subtracted out. However in future generations of the system, this should be eliminated.

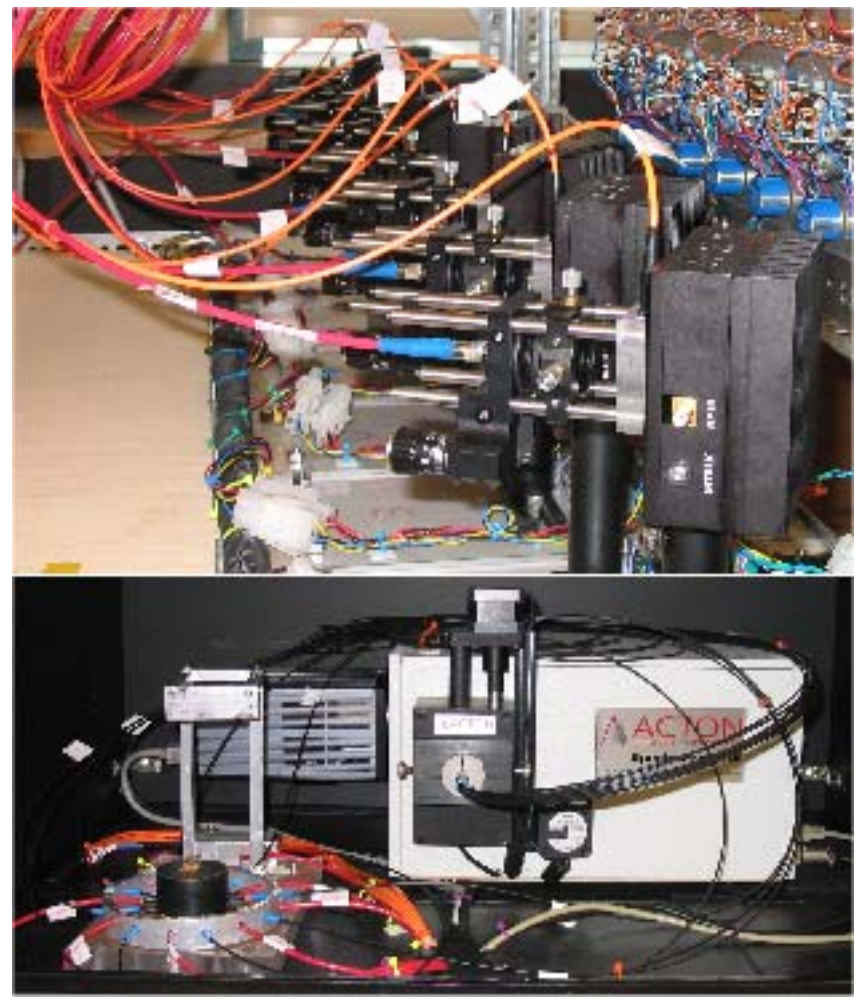

Figure 2. The eight diode lasers are shown with fiber temperature controlled laser (upper photo) and the fibers feed into the phantom/tissue imaging array shown in the lower photo, at left The black pick up fibers exit the imaging region, and are all bundled into the spectrometer in the center of the photo, at the linear entrance slit. The Acton spectrometer spreads the wavelengths with a $1200 \mathrm{l} / \mathrm{mm}$ grating, onto the video rate capable CCD, Cascade 512F (Princeton Instruments).

Using homogeneous phantoms, the intensity was plotted as a function of distance between the source and detector point, as a way to determine if the attenuation followed that which would be expected for a diffusing medium. This log intensity plot is shown in Figure 3(b), along with the best fit exponential decay curves. The straight lines indicate that the attenuation was approximately like a direct exponential curve, with the slope of this graph being the effective attenuation coefficient.
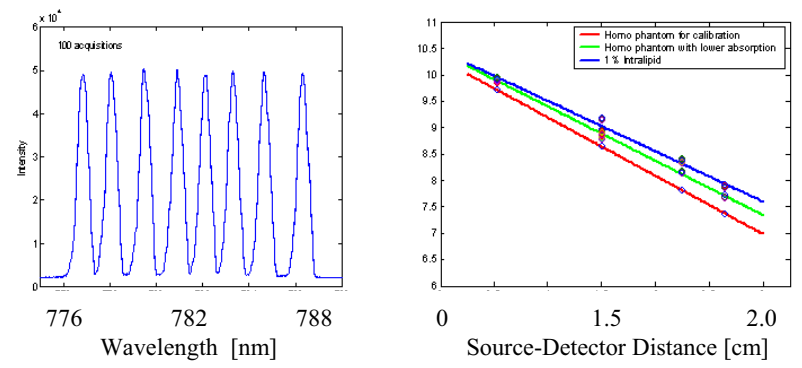

Figure 3. In (a), the readout of equal intensity input from all eight lasers is shown with the wavelength listed along the bottom, to illustrate the distribution of wavelengths used. In (b) the output 
intensity as a function of distance from the source is listed for three different imaging regions.

The photograph shown in Figure 4 shows the set up for phantom testing of dynamic changes, with a cup of Intralipid and a black ball bearing being rotated within the cup. The path of rotation is illustrated in the diagrams at right and at bottom. Once again, the CCD readout was streamed to file and the intensities extracted for post processing. NIRFAST [9] image reconstruction was used to recover images, with assumed transport scattering coefficients, as predicted by diffusion theory. Reconstructed images of the ball bearing rotating are shown in Figure 5. Interestingly, there are small intensity fluctuations in the reconstruction as the ball passes between the adjacent fibers, but the overall reconstruction repeatability with position was within $15 \%$.

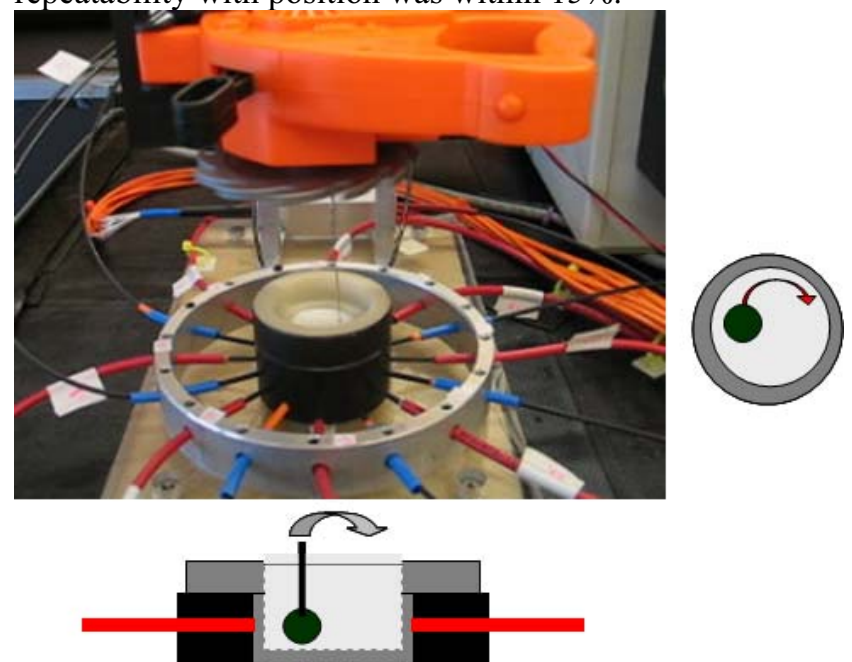

Figure 4. A photogram of the phantom is shown with Intralipid in the imaging volume, and a small wire holding a black ball bearing which was rotated using a circular rotary tool.

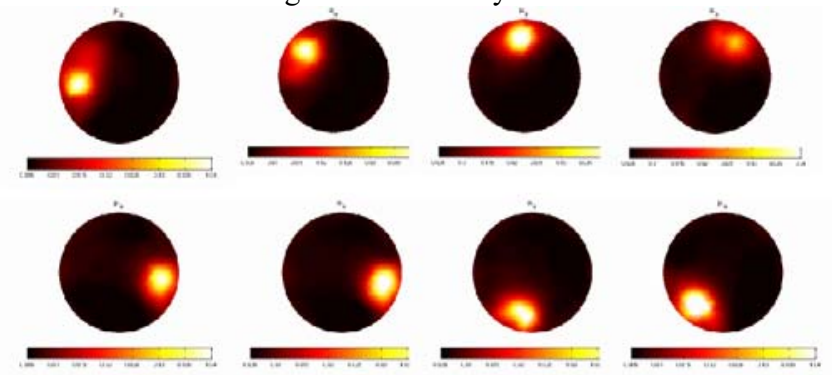

Figure 5. Tomographic images of the rotating ball bearing are shown for single successive frames.

For initial in vivo testing, a mouse leg was utilized to image the blood pulsation in the muscle. Both legs were imaged successively, with one leg having compromised blood flow due to blockage of the iliac artery, while the other leg had normal blood flow. The tomographic images of these legs are shown below in Figure 6, with each pair of images being one of a sequence of images at $1 / 30^{\text {th }}$ of a second. The blood flow pulsation in the left leg can be seen between images 3 and 4 as a successive decrease in overall intensity, whereas the intensity of pulsation is significantly less in the right leg.

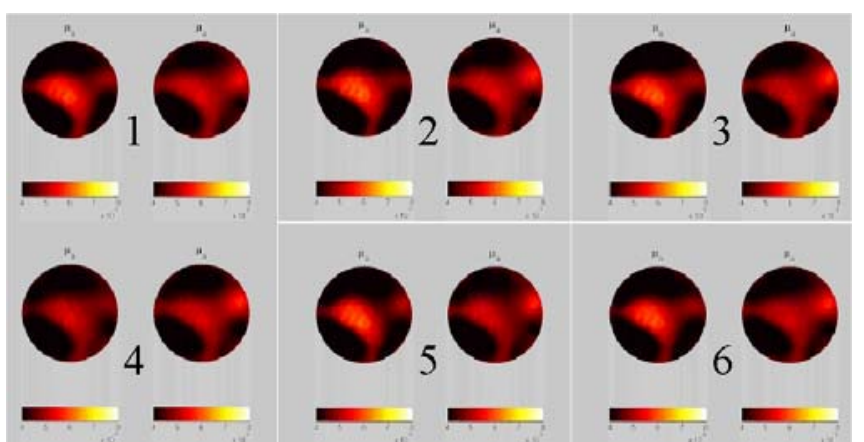

Figure 6. Tomographic images of the two legs of a mouse are shown, at successive time points, with 1 frame every $1 / 30^{\text {th }}$ of a second. The leg at right in each pair of frames was normal and the leg at left in each pair of images had the iliac artery blocked. The lower overall absorption coefficient is apparent in the leg at left, and the pulsation in the leg at right is more apparent in between frames 3, 4 and 5. This pulsation is due to blood flow. The imaging area is $27 \mathrm{~mm}$ diameter, filled with Intralipid, and the leg is placed into the intralipid. The color bar scales from 0.004 to $0.008 \mathrm{~mm}^{-1}$ in absorption coefficient.

\section{DISCUSSION}

The system tested and illustrated here was described in detail recently in two publications $[6,7]$, but the data from the system is further illustrated here with in vivo testing. The system provides capture of tomographic data at 35 frames per second at a single wavelength band. Alterations are ongoing to add a second wavelength band to the system, to allow capture of oxygen saturation and total hemoglobin images.

Dynamic range issues limit the current configuration to small animal imaging, yet extension to larger animals is possible with careful optimization of the laser sources and CCD intensity calibration and attenuation. Ongoing studies illustrate that frequency domain detection is also possible by replacing the CCD with a fiber coupler to PMTs. PMTs have a faster temporal response which would allow detection of signals in the range of $100 \mathrm{MHz}$, and they also have a lower power detection threshold. This modified configuration will be demonstrated in the near future. 
This project has been funded by NIH grant R21CA100984,

and used resources from P01CA080139 and

U54CA105480.

\section{REFERENCES}

1. Zhu, Q., S.H. Kurtzma, P. Hegde, S. Tannenbaum, M. Kane, M. Huang, N.G. Chen, B. Jagjivan, and K. Zarfos, Utilizing optical tomography with ultrasound localization to image heterogeneous hemoglobin distribution in large breast cancers. Neoplasia, 2005. 7(3): p. 263-70.

2. Li, A., E.L. Miller, M.E. Kilmer, T.J. Brukilaccio, T. Chaves, J. Stott, Q. Zhang, T. Wu, M. Choriton, R.H. Moore, D.B. Kopans, and D.A. Boas, Tomographic optical breast imaging guided by three-dimensional mammography. Applied Optics, 2003. 42(25): p. 5181-5190.

3. Brooksby, B., S. Jiang, H. Dehghani, B.W. Pogue, K.D. Paulsen, C. Kogel, M. Doyley, J.B. Weaver, and S.P. Poplack, Magnetic Resonance-Guided Near-Infrared Tomography of the Breast. Rev. Sci. Instr., 2004. 75(12): p. 5262-5270.

4. Brooksby, B., S. Jiang, H. Dehghani, B.W. Pogue, K.D. Paulsen, J.B. Weaver, C. Kogel, and S.P. Poplack, Combining near infrared tomography and magnetic resonance imaging to study in vivo breast tissue: implementation of a Laplacian-type regularization to incorporate MR structure. J. Biomed. Opt., 2005. 10(5): p. 050504-1-10.

5. Brooksby, B., S. Srinivasan, S. Jiang, H. Dehghani, B.W. Pogue, K.D. Paulsen, J. Weaver, C. Kogel, and S.P. Poplack, Spectral-prior information improves Near-Infrared diffuse tomography more than spatial-prior. Opt. Lett., 2005. 30(15): p. 1968-1970.

6. Piao, D., S. Jiang, S. Srinivasan, H. Dehghani, and B.W. Pogue, Video-rate near-infrared optical tomography using spectrally-encoded parallel light delivery. Opt. Lett., 2005. 30(19): p. 2593-2595.

7. Piao, D., H. Dehghani, S. Jiang, S. Srinivasan, and B.W. Pogue, Instrumentation for video-rate nearinfrared diffuse optical tomography. Rev. Sci. Instr., 2005. 76: p. 124301.

8. Franceschini, M.A., K.T. Moesta, S. Fantini, G. Gaida, E. Gratton, H. Jess, W.W. Mantulin, M. Seeber, P.M. Schlag, and M. Kaschke, Frequencydomain techniques enhance optical mammography: initial clinical results. Proc. Nat. Acad. Sci USA, 1997. 94(12): p. 6468-73. 